\title{
Using a computer simulation to teach attitude surveying
}

\author{
MALCOLM J. GRANT \\ Memorial University of Newfoundland \\ St. John's, Newfoundland, Canada
}

\begin{abstract}
An interactive computer program that simulates, for teaching purposes, the procedures involved in carrying out an attitude survey is described. Students make decisions about the content and length of an attitude questionnaire, as well as about the procedures to be used to sample the population. Students' inferences about the population characteristics can be compared with the actual characteristics (predetermined by the instructor) to provide students with an indication of the merits of the alternatives they chose.
\end{abstract}

Within the last decade, a number of attempts have been made to employ computer simulations for instructional purposes in undergraduate laboratory courses (see, e.g., Eamon, 1980, Fazio \& Backler, 1983, and Forbach, 1979). The advantages of the simulation approach over traditional laboratory teaching methods were well documented by Thurmond and Cromer(1975). The present paper describes a computer simulation program (ATTSIM) that we have designed to give students in our social psychology laboratory course some experience in the decision-making processes involved in carrying out an attitude survey.

Students in the laboratory interact with a VAX-11/780 computer; they make a series of decisions about the length and content of an attitude questionnaire, the method of obtaining a list of the population, the size of the sample to be drawn, the method of contacting respondents, and the number of "call-backs" to be made in cases in which respondents are difficult to contact. Questionnaire data are generated by ATTSIM in accordance with a normally distributed population of attitude scores. Students then analyze the data they have obtained and attempt to infer the population characteristics. Comparisons between the inferred and actual population characteristics (predetermined by the instructor) can provide students with an indication of the biases introduced by the alternatives they chose.

\section{DESCRIPTION OF THE SIMULATION}

\section{Constructing the Questionnaire}

Before the simulation is run, a large pool of possible questionnaire items is generated either by the instructor

The author wishes to thank Ted Hannah for valuable suggestions concerning the uses of the simulation described in this paper. The author's mailing address is: Department of Psychology, Memorial University of Newfoundland, St. John's, Newfoundland, Canada A1B 3X9. or by the students working together. The instructor privately assesses each item in terms of (1) its probable validity (i.e., whether or not responses to that item are likely to reflect the respondent's true attitude score), (2) its probable susceptibility to social desirability bias, and (3) its probable error variability.

Validity, bias, and error scores for each item are stored together with the items themselves in data statements in ATTSIM. During the simulation, items are displayed one at a time and the student is asked to decide whether or not he/she wishes to include that particular item on the questionnaire. It should be noted that students run a compiled version of ATTSIM and thus are prevented from reading the contents of the program itself.

\section{Listing the Population}

In the current version of ATTSIM, students are given a choice among three alternative lists for a city population: the phone book, the voters' list, and the city directory. The instructor assigns a bias factor (any positive or negative number) to each of these three methods. This bias factor is added to the mean of the population distribution of attitude scores from which the student samples. In addition to a bias factor, the instructor assigns a single cost factor to each list method; this factor represents the amount of time, effort, and money that the use of such a method would involve in a real survey. Although, in principle, time, effort, and money are separate costs and could be represented as such, a single cost factor has been used to represent all three aspects in the current simulation. This is admittedly an oversimplification, but it is not entirely unrealistic. Obtaining a complete and up-to-date listing of any population typically involves considerable expenditure of all three resources.

The cost factor is translated into a time delay by a function in ATTSIM. In general, the methods that are most effective in minimizing bias require that the stu- 
dent spend a longer period of time at the terminal as he or she waits for the computer to respond. For example, using a phone book to draw a sample of 100 respondents from a population causes a delay for the student of about $1.5 \mathrm{~min}$. Using a city directory (in which living quarters and adults wtihin living quarters are listed) to draw a similarly sized sample results in a delay of approximately $5 \mathrm{~min}$. This is only the first of several such delays that the persistent student must endure in order to obtain valid data. There are, of course, a number of other ways in which the notion of costs could be operationalized in the simulation, but we have found that the inclusion of the time-delay function appears to give our students an appreciation of some of the more practical constraints under which pollsters in the real world must frequently operate.

\section{Drawing the Sample}

Students are asked to decide how large a sample they wish to draw. The main advantage of a large sample, of course, has to do with the precision of the results obtained. For the student, the main disadvantage of drawing a very large sample lies in the amount of time required both to gather the data and later to analyze the data. After the student has exhausted the original sample (and perhaps been dismayed by the low completion rate), he or she may draw a further sample.

Once the sample has been drawn, ATTSIM generates three scores for each respondent: an attitude score, a social desirability or bias score, and a "willingness-tocooperate" score. All of these scores are assumed to be normally distributed in the population, and the means and standard deviations are specified in advance by the instructor. The attitude and social desirability scores are used together to determine the respondent's answers to particular questionnaire items, and the willingness score becomes a partial determinant of whether or not data are obtained from a particular respondent.

\section{Contacting Respondents}

The three most common methods of contacting respondents-by phone, by mail, and in a door-to-door interview - have been incorporated in ATTSIM. Students are asked to choose one of these methods. The most important consequence of this decision involves the different amounts of time that the contact methods require. The time-delay function in ATTSIM ensures that the contact methods that are most effective in obtaining respondents cost more in terms of the student's time. The cost factor for each contact method can be varied by the instructor.

The student attempts to contact each simulated respondent, in turn, by using the method he or she has chosen. On each attempt, a contact score (c) is computed according to the equation $c=(a s+b r) /(|a|+b)$. In this equation, $s$ is the respondent's standardized attitude score, $r$ is a normally distributed and standardized random variate, and $a$ and $b$ are weighting factors provided by the instructor. Once calculated, the contact score is compared with a criterion score provided by the instructor. Only if the contact score exceeds this criterion score will the contact attempt be successful. Thus, the instructor can adjust the overall contact rate by varying the size of the criterion score.

Because the probability of contacting a particular respondent is partly a function of that respondent's attitude score, an element of bias is introduced. People in a sample who are particularly difficult to contact (e.g., those who work very long hours) may have attitudes somewhat different from those of people who can be contacted more easily. The instructor determines the direction and seriousness of this source of bias in the simulation by adjusting the values of $a$ and $b$ in the contact-score equation. If $\mathrm{a}$ is positive, respondents with high attitude scores will be more likely to be contacted; if a is negative, respondents with low attitude scores will be more likely to be contacted.

Unlike the respondent's attitude score, which remains fixed, the random factor, reflecting situational influences, is assumed to vary with each contact attempt. Thus, a respondent who cannot be contacted on one occasion may be contacted on the next. Students can make as many or as few contact attempts as they wish. Persistence in this regard increases the number of respondents contacted and thereby reduces sample bias.

\section{Obtaining Consent}

In the simulation, a respondent who has been contacted may or may not consent to answer the questionnaire. For each respondent who is contacted, a consent score is computed according to an equation similar in form to that used in computing the contact score. The consent score is assumed to be a weighted function of (1) the respondent's attitude score, (2) the respondent's willingness-to-cooperate score, (3) the contact method used, (4) the length of the questionnaire, and (5) a random factor. The instructor determines the weight to be given to each of these five factors. If the consent score exceeds a criterion score assigned by the instructor, and if the respondent's willingness score exceeds some minimum value, the respondent will agree to complete the questionnaire; otherwise, the respondent will refuse.

As noted above, the consent probability is partly a function of characteristics of the respondent (i.e., his or her attitude and willingness) and partly a function of situational factors (i.e., how the person is contacted and the length of the questionnaire). The inclusion of the respondent's attitude as a partial determinant of the consent probability introduces an additional source of bias to the eventual sample. Those who consent and those who do not may differ somewhat in terms of the attitudes being assessed. The inclusion of the willingness determinant introduces no particular bias but does add a realistic element of within-person consistency (i.e., some people will refuse to respond to surveys regardless of when or how they are approached).

Unlike the respondent characteristics that influence the consent probability, the situational influences can be 
controlled by the student. The choice of certain contact methods (e.g., the door-to-door interview) typically maximizes the respondent consent rate, whereas the choice of other contact methods (e.g., mail surveys) most often results in a low consent rate. Similarly, the length of the questionnaire may be a factor in whether or not a respondent consents. Thus, a student who includes a large number of items on his or her questionnaire may pay a penalty in terms of a somewhat lower consent rate.

Finally, a random factor in the computation of the consent score has been included to summarize the variety of other person and situation factors that may play a role in determining whether a particular respondent will consent on a particular occasion.

\section{Producing the Data}

Once a simulated respondent has agreed to complete the questionnaire, ATTSIM generates a score for that respondent on each of the items that the student has included on the questionnaire. A respondent's item score is assumed to be a function of that respondent's attitude (if the item is valid), the respondent's social desirability motivation (if the item is biased), and, finally, the error variability of the item. Once the item scores have been computed, they are displayed one at a time, together with the appropriate item on the student's terminal. It is left to the student to record the data and to carry out the analyses necessary to infer the population characteristics, keeping in mind the direction in which each item is worded. It would, of course, be a simple matter to have ATTSIM write the data directly to a disk file that could be accessed later by some statistical package. It has been our experience, however, that students who have had relatively little experience with data gathering and computers can easily lose sight of the meaning of the data when its collection and processing are entirely automated.

\section{USING THE SIMULATION}

One approach in using ATTSIM is to have students carry out a series of single-factor experiments in which they are told to hold constant all factors except one (e.g., the number of call-backs, the size of the sample, or the length of the questionnaire). In this way, the effect of each of these variables can be clearly demonstrated. This approach is likely to be most effective with students who have had relatively little experience with the basic concepts of sampling and survey work.

A less structured approach can sometimes be effective with more sophisticated students. Such students can be left on their own to experiment with ATTSIM, as they try a variety of alternatives and compare the results. In a class meeting later, the data that each student has generated and his or her best estimate of the population characteristics (predetermined by the instructor) can be used as a basis for discussion of such concepts as reliability, validity, sampling bias, and error variability.
ATTSIM has been used with 3rd-year social psy. chology majors who have completed two one-semester courses in statistics and research design. A small number of these students have completed computer science courses. With these students, we have usually taken an approach that combines aspects of the approaches desribed above.

\section{LANGUAGE AND COMPUTER}

ATTSIM is a 300 -line program written in BASIC for a VAX-11/780 computer. This version of BASIC has certain characteristics that might necessitate some routine editing before ATTSIM is run on other systems (e.g., lines with multiple statements separated by back slashes). In addition, the program contains a number of screen- and cursor-control PRINT statements that are specific to the Digital Equipment Corporation VT101 terminals used in our laboratory. These must be modified in accordance with the particular video terminals being used.

Two features of ATTSIM facilitate its modification by individual instructors. First, all the parameters that control the costs and the direction and extent of the various biases are given values in the first 30 statements of the program. Each of these statements is identified with an appropriate comment on the same line. Second, the survey items (currently a set of 30 statements deal. ing with the death penalty) are stored in data statements with validity, bias, and error scores, close to the end of the program. These statements can be easily modified or replaced without altering the rest of the program in any way. The number of survey statements used might need to be reduced if the program were to be run on a system with less than $48 \mathrm{~K}$ of memory.

\section{AVAILABILITY}

A complete listing of ATTSIM and a 28-page instructor's manual giving details concerning calculations within ATTSIM and the setting of parameter values are available without charge. Send requests to Malcolm J. Grant, Department of Psychology, Memorial University of Newfoundland, St. John's, Newfoundland, Canada A1B $3 \times 9$.

\section{REFERENCES}

EAmon, D, B. (1980). LABSIM: A data-driven simulation program for instruction in research design and statistics. Behavior Research Methods \& Instrumentation, 12, 160-164.

Fazio, R. H., \& Backlen, M. H. (1983). Computer lessons for a social psychology research methods course. Behavior Research Methods \& Instrumentation, 15, 135-137.

Forbach, G. B. (1979). EXPER SIM: Review and update. $B e$ havior Research Methods \& Instrumentation, 11, 519-522.

Thurmond, J. B., \& Cromer, A. D. (1975). Models and modeling with the Louisville Experiment Simulation System (LESS). Behavior Research Methods \& Instrumentation, 7, 229-232.

(Manuscript received August 4, 1983; revision accepted for publication December 2, 1983.) 